\title{
Preoperative and Postoperative Management of Arthroscopic Rotator Cuff Repair
}

\section{ISSN: 2576-8875}

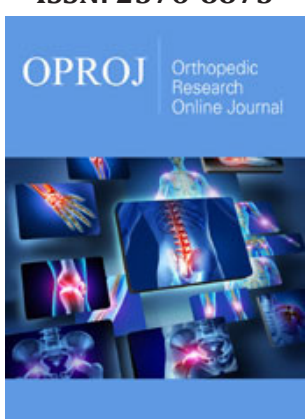

For HTML Version scan this QR code:

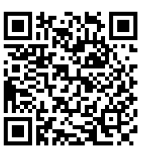

${ }^{* 1}$ Corresponding author: Hisahiro Tonotsuka, Department of Orthopaedic Surgery, Kanagawa Rehabilitation Hospital, 516 Nanasawa Atsugi Kanagawa, 243-0121, Japan

Submission: 非 February 27, 2019

Published: 眥March 06, 2019

Volume 5 - Issue 2

How to cite this article: Hisahiro T. Preoperative and Postoperative Management of Arthroscopic Rotator Cuff Repair. Ortho Res Online J. 5(2). OPROJ.000609.2019.

DOI: 10.31031/OPROJ.2019.05.000609

Copyright@ Hisahiro Tonotsuka, This article is distributed under the terms of the Creative Commons Attribution 4.0 International License, which permits unrestricted use and redistribution provided that the original author and source are credited.

\author{
Hisahiro Tonotsuka ${ }^{1,3 *}$, Hiroyuki Sugaya ${ }^{2}$, Norimasa Takahashi ${ }^{2}$, Nobuaki \\ Kawai $^{2}$, Hajime Sugiyama ${ }^{1,3}$, Keishi Marumo ${ }^{3}$ \\ ${ }^{1}$ Department of Orthopaedic Surgery, Kanagawa Rehabilitation Hospital, Japan \\ ${ }^{2}$ Funabashi Orthopaedic Sports Medicine Center, Japan \\ ${ }^{3}$ Department of Orthopaedic Surgery, The Jikei University School of Medicine, Japan
}

Opinion

Considerable advances have been made in arthroscopic rotator cuff repair (ARCR) over the last decade and excellent results have been achieved. Although recent studies have shown that functional outcomes after ARCR are comparable with those after open or miniopen repair, further refinements that could improve the clinical outcome of ARCR have been widely debated. Various factors can affect the clinical results, some medical, such as operative technique and preoperative and postoperative management, and others patient-related, such as tear size and the presence of diabetes or preoperative contracture. Though efforts have been focused on resolving the medical factors, patient-related factors should also be addressed.

A number of complications of ARCR have been reported, one of the most common of which is persistent shoulder stiffness. Although loss of shoulder mobility after ARCR is widely recognized, the relationship between clinical management and shoulder stiffness is poorly understood. We believe that appropriate preoperative management and postoperative rehabilitation are as important as surgical technique and should be able to achieve good results.

\section{Preoperative management}

Preoperative contracture is one of the factors affecting the final result of rotator cuff repair. The frequency of preoperative contracture has been reported to be $10 \%-25 \%$, although the definitions used have not been consistent [1-5]. Persistent limitation of range of motion (ROM) after ARCR is more likely in patients with preoperative contracture than in those without [2]. Several investigators have recommended methods for improving preoperative stiffness in patients with rotator cuff tear, including improvement of ROM by preoperative rehabilitation before ARCR [3], intraoperative manipulation [4], and simultaneous arthroscopic release of the capsule if the contracture is severe [5]. Each of these methods has been reported to have good results.

On the other hand, many patients have both daytime and nighttime aching pain that disturbs sleep and requires anti-inflammatory medication. Patients with spontaneous pain before surgery often continue to have pain after surgery, which can impede effective rehabilitation. Some studies suggest that this pain is related to clinical inflammation in the shoulder joint or bursa [6,7]. Intra-articular corticosteroid injections [8] and injections into the subacromial bursa [9] have been reported to be effective in patients who have this type of inflammatory pain preoperatively, control of which affects the final outcome [10]. Therefore, preoperative inflammatory pain should be managed appropriately, while taking into account the risks of infection associated with corticosteroid injections and structural failure.

\section{Postoperative management}

It is widely known that rehabilitation in the early stage after ARCR has a favorable effect on the clinical outcome. Although Koo et al. [11] suggested that inappropriate immediate postoperative care can result in postoperative contracture [11], there is ongoing debate about 
the postoperative treatment protocol. Some authors recommend immediate passive motion exercise starting immediately after surgery [12] while others have reported that 6 weeks of immobilization produces better results than immediate passive motion [13]. However, shorter period of immobilization and excessive rehabilitation may increase the risk of postoperative structural failure. A histological study in a primate model by Sonnabend et al. [14] found that a considerable number of Sharpey fibers appeared to be healed at 8 weeks and that the bone and tendon were held together by 12-15 weeks. They suggested that the rehabilitation protocol in humans should be mild for at least 1215 weeks after surgery to allow sufficient tendon-to-bone healing to protect the surgical repair [14]. We have reported that $120^{\circ}$ of forward flexion and $20^{\circ}$ of side-lying external rotation should be the minimum target ROM at 3 months after surgery, regardless of whether patients undergo active rehabilitation or immobilization [15]. Our experience has been that the outcome at 2 years postoperatively is less favorable in patients who do not reach this ROM target than in those who do, but that there is no significant difference in the re-tear rate. Appropriate rehabilitation is important for achieving this target ROM and attaining good results. Forced exercise and excessive motion that produces pain should be avoided. Physiotherapy should be performed to mitigate the tonus of the outer muscles and promote flexibility of the shoulder girdle, including the muscles around the scapula, ribs, and thoracic vertebrae. Physiotherapy should then progress to include circular movement of the shoulder joint while reducing trick movements. We consider that performing appropriate physiotherapy is essential, especially in the early stage after surgery.

In summary, it is desirable to improve the patient's ROM and to manage inflammatory pain to the extent possible before performing ARCR. If the ROM cannot be improved preoperatively, additional treatment is required. Appropriate postoperative rehabilitation is also necessary. Training to a level that causes pain should be avoided.

\section{Conflict of Interests}

The author declare no potential conflicts of interest with respect to the research, authorship, and/or publication of this article.

\section{References}

1. Hsu SL, Ko JY, Chen SH, Wu RW, Chou WY, et al. (2007) Surgical results in rotator cuff tears with shoulder stiffness. J Formos Med Assoc 106(6): 452-461.
2. Tauro JC (2006) Stiffness and rotator cuff tears: incidence, arthroscopic findings, and treatment results. Arthroscopy 22(6): 581-586.

3. Matsen FA, Titelman RM, Lippitt SB, Wirth MA, Rockwood CA, et al. (2004) The shoulder. Saunders Co, Rotator cuff, Philadelphia, USA, 3: 795-878.

4. Cho NS, Rhee YG (2008) Functional outcome of arthroscopic repair with concomitant manipulation in rotator cuff tears with stiff shoulder. Am J Sports Med 36(7): 1323-1329.

5. Cho CH, Jang HK, Bae KC, Lee SW, Lee YK, et al. [2015] Clinical outcomes of rotator cuff repair with arthroscopic capsular release and manipulation for rotator cuff tear with stiffness: a matched-pair comparative study between patients with and without stiffness. Arthroscopy 31(3): 482487.

6. Okamura K, Kobayashi T, Yamamoto A, Shitara H, Osawa T, et al. (2017) Shoulder pain and intra-articular interleukin-8 levels in patients with rotator cuff tears. Int J Rheum Dis 20(2): 177-181.

7. Santavirta S, Konttinen YT, Antti-Poika I, Nordström D (1992) Inflammation of the subacromial bursa in chronic shoulder pain. Arch Orthop Trauma Surg 111(6): 336-340.

8. Weiss JJ (1981) Intra-articular steroids in the treatment of rotator cuff tear: reappraisal by arthrography. Arch Phys Med Rehabil 62: 555-557

9. Shin SJ, Do NH, Lee J, Ko YW (2016) Efficacy of a subacromial corticosteroid injection for persistent pain after arthroscopic rotator cuff repair. Am J Sports Med 44(9): 2231-2236.

10. Tonotsuka H, Sugaya H, Takahashi N, Kawai N, Sugiyama H, et al. (2019) Preoperative pain control in arthroscopic rotator cuff repair: Does it matter? Clin Orthop Surg 11: e25.

11. Koo SS, Parsley BK, Burkhart SS, Schoolfield JD (2011) Reduction of postoperative stiffness after arthroscopic rotator cuff repair: Results of a customized physical therapy regimen based on risk factors for stiffness. Arthroscopy 27(2): 155-160.

12. Arndt J, Clavert P, Mielcarek P, Bouchaib J, Meyer N, et al. (2012) Immediate passive motion versus immobilization after endoscopic supraspinatus tendon repair: a prospective randomized study. Orthop Traumatol Surg Res 98(6 Suppl): S131-138.

13. Keener JD, Galatz LM, Stobbs-Cucchi G, Patton R, Yamaguchi K (2014) Rehabilitation following Arthroscopic rotator cuff repair: a prospective randomized trial of immobilization compared with early motion. J Bone Joint Surg Am 96(1): 11-19.

14. Sonnabend DH, Howlett CR, Young AA (2010) Histological evaluation of repair of the rotator cuff in a primate model. J Bone Joint Surg Br 92(4): 586-594.

15. Tonotsuka H, Sugaya H, Takahashi N, Kawai N, Sugiyama H, et al. (2017) Target range of motion at 3 months after arthroscopic rotator cuff repair and its effect on the final outcome. J Orthop Surg 25(3): 1-8. 